\title{
Assessment of physical activity status among pregnant women in southwestern China
}

Original article

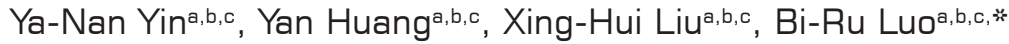

aWest China Second University Hospital, Sichuan University, Chengdu, Sichuan 610041, China

${ }^{b}$ Key Laboratory of Birth Defects and Related Diseases of Women and Children, Sichuan University, Ministry of Education, Chengdu, Sichuan 610041, China

'Department of Gynecology, Sichuan University, Chengdu, Sichuan 610041, China

Received: 8 September 2018; Accepted: 30 November 2018; Published: 20 June 2019

Abstract: Objective: To assess the patterns and intensities of physical activity among pregnant women in southwestern China, which help us create a fitness regimen based on the 2002 American College of Obstetricians and Gynecologists (ACOG) guidelines for exercise during pregnancy.

Methods: A cross-sectional study was performed to recruit pregnant women at a prenatal checking visit clinic with the self-administered Chinese version of Pregnancy Physical Activity Questionnaire (PPAQ).

Results: A total of 1179 Chinese pregnant women were enrolled, of whom $92.60 \%$ were classified as not meeting the ACOG guidelines. By intensity, $47.36 \%$ of the score was attributed to light-intensity activities ( 1.5 to $<3.0$ metabolic equivalent [MET]) in the first trimester; the other 2 trimesters were similar. By patterns, about $45.15 \%$ of the score was attributed to inactivity. More than $82.35 \%$ of enrolled pregnant women regarded slowly walking as the most common type of exercise during pregnancy.

Conclusions: This study represents a group of Chinese women who maintained an inactive lifestyle, including low-intensity activities and unitary type of exercises during their pregnancy. Health-care providers are advised to provide appropriate physical activity guidelines to pregnant women. Development of MET-hours/week recommendations is warranted to promote greater physical activity during pregnancy.

Keywords: physical activity • cross-sectional studies • pregnancy • inactive lifestyle • metabolic equivalent, MET

(c) Shanxi Medical Periodical Press.

\section{Introduction}

Since early years of this century, the benefits of exercise during pregnancy have been realized and pregnant women have been encouraged to do regular physical activity in the absence of medical or obstetric complications. ${ }^{1}$ Studies have reported that physical activity during pregnancy may reduce the risk of pregnancy complications such as excessive gestational weight gain, ${ }^{2,3}$ gestational diabetes, ${ }^{4,5}$ preeclampsia, ${ }^{6}$ and cesarean delivery. ${ }^{7}$ In the acknowledgment of these benefits, several physical organizations endorse

How to cite this article: Yin Y-N, Huang Y, Liu X-H, Luo B-R. Assessment of the physical activity status among pregnant women in southwestern China. Front Nurs. 2019; 2: 1-7. 
physical activity during pregnancy with position statement. In 2002, the American College of Obstetricians and Gynecologists (ACOG) recommended that pregnant women should exercise at moderate intensity for at least 30 minutes on most, if not all, days of the week if no medical or obstetric complications are present. ${ }^{8}$ In addition, the US government included a section on pregnancy recommendations in its national "2008 Physical Activity Guidelines for Americans." However, pregnancy is a period in the life of women which is often associated with decreased participation in sports and exercise,${ }^{10}$ and the majority of women fail to meet the ACOG physical activity guidelines for pregnancy. ${ }^{8}$ Ning et al. ${ }^{11}$ reported that $23 \%$ of previously active women ceased to engage in exercise completely during pregnancy.

China has more than 10 million live births every year. ${ }^{12}$ Since implementation of the "one child" policy, pregnant women in China became the focus of family's attention, and their housework and occupational workload have been reduced considerably. ${ }^{12}$ At the same time, pregnant women spend more and more time on sedentary activities, ${ }^{13}$ which leads to many negative pregnancy-related outcomes such as high incidence of gestational diabetes, macrosomia, and cesarean section. ${ }^{14}$ However, the studies of physical activity during pregnancy on the assessment of recreational activity among populations of predominantly Chinese women were sparse and there were no established physical activity guidelines for Chinese pregnant women. So, it is crucial to assess the physical activity level among Chinese pregnant women to provide appropriate sports guidelines.

The main objectives of the present study were to (1) assess the changes in patterns, intensities, and frequencies in physical activity levels during pregnancy using the Chinese version of Pregnancy Physical Activity Questionnaire (PPAQ) and (2) provide baseline measurements of physical activity during pregnancy for further studies.

\section{Methods}

\subsection{Study population and design}

A cross-sectional study was performed to collect information using the Chinese version of the PPAQ. Recruitment began in October 2013. A total of 1188 eligible pregnant women were enrolled (396 in the first trimester, 396 in the second trimester, and 396 in the third trimester) when they took prenatal checkup in the West China Second University Hospital of Sichuan University. Women were considered ineligible for the survey if they had any of the following characteristics: ${ }^{15}$ diabetes requiring insulin, hypertension or heart disease requiring medication, chronic renal disease, nonsingleton pregnancy, and under 16 or over 40 years of age. Interviewers informed patients of the aims and procedures of the study, and each patient read and signed a written informed consent. The physical activity assessment was self-administered using the modified PPAQ; patients in the first trimester were about 12 weeks pregnant, the second trimester were between 24 and 28 weeks, and the third trimester were more than 32 weeks.

\subsection{Physical activity assessment using the PPAQ}

The majority of currently available physical activity questionnaires have been developed and validated in men, and most fail to include household or childcare activities. ${ }^{16}$ Here, we have considered the PPAQ for its design, with the aim of measuring the physical activity during pregnancy and for its development based on data collected among prenatal care patients. ${ }^{15}$ The PPAQ provides a semiquantitative measure of a wide range of physical activity patterns and intensities.

Chasan-Taber, one of the original authors of the PPAQ, granted permission for the development and use of a Chinese version of the PPAQ. For the purpose of this study, the PPAQ was translated into Chinese and tested for the acceptability of the wording. Since parts of the PPAQ were found inappropriate for Chinese pregnant women, for instance, some items are related to playing with pets, or mowing lawn, which rarely occur among the new generation of Chinese pregnant women. ${ }^{12}$ So, we made some changes to the questionnaire, such as removing these 2 items and adding "Going up and down stairs" to the domain of sports/exercise, because it is common among Chinese pregnant women. The energy expenditure of the new items was calculated by metabolic equivalent (MET) according to the Compendium of Physical Activities Tracking Guide. ${ }^{17}$ The content, technical, conceptual, semantic, and experiential equivalents of cultural adaptation were discussed by the research members and experts at each procedure. After crosscultural adaptation, the Chinese version of the PPAQ is suitable for the present study.

The last version of the questionnaire contains 5 domains of physical activities including "Household/ Care-giving" (9 activities), "Occupational" (5 activities), "Sports/Exercise" (8 activities + 2 open-ended), "Transportation" (3 activities), and "Inactivity" (4 activities). Women were categorized according to whether they achieved ACOG guidelines. Women with $\geq 10$ METhours/week in sports/exercise activities of moderate intensity or greater were considered to have met the ACOG guidelines. ${ }^{18}$ 
The Chinese version of the PPAQ was self-administered and took about 15 minutes to complete. The weekly average of energy expenditure (MET-hours/ week) was obtained by the reported time spent at each activity multiplied by its intensity and summed to derive the weekly score. The intensities were grouped into 4 categories: sedentary activity $(<1.5 \mathrm{MET})$, light intensity $(1.5$ to $<3.0 \mathrm{MET})$, moderate intensity $(\geq 3.0$ to $\leq 6.0$ $\mathrm{MET})$, and vigorous intensity (>6.0 MET).

\subsection{Pretest}

To validate the Chinese version of the PPAQ, we recruited 60 subjects from Chengdu, located in southwestern China, via prenatal care patients in West China Second University Hospital of Sichuan University. According to the reflection of the opinions of pregnant women, we revised the Chinese version of the questionnaire and derived the final version of the PPAQ.

\subsection{Statistical and data analysis}

All analyses were conducted with SAS9.1 (Raleigh, NC). For the continuous or ordinal measurements (e.g., maternal age and PPAQ scores), proportion, quartile, means, and variations (SD) were calculated to describe the sociodemographic characteristics, lifestyle habits, and results of the survey. $p$-values for the comparison of the 3 trimesters were assessed with ANOVA for continuous variables, the chi-square tests, Kruskal-Wallis test, and Fisher's exact test, in case of small cell size, for categorical variables. Cronbach's a coefficient and content validity were used to evaluate the reproducibility of the Chinese version of the PPAQ.

\section{Results}

\subsection{Participant characteristics}

A total of 1179 pregnant women completed the survey from October 2013 to January 2014. Among them, 391, 392, and 396 women were in the first, second, and third trimesters, respectively. Overall, the majority of participants were Han (95.65\%-97.73\%), nulliparous (76.79\%-79.29\%), employed or studying $(72.70 \%-76.01 \%)$, and living in city $(77.81 \%-84.85 \%)$ medium per capita income (over $36000 ¥$, from $81.89 \%$ to $86.36 \%$ ) and high level of education (some college/ graduate from $52.29 \%$ to $64.65 \%$ ). There was no statistically significant difference between the ages, prepregnancy body mass indexes (BMls), annual per capita incomes, cigarette smoking, and occupational status in different trimesters (Table 1).

\subsection{Self-reported measures of physical activity using the PPAQ}

Cronbach's a coefficient and content validity were 0.76 and 0.93 , respectively. A total of $5.64 \%$ in the first trimester, $7.40 \%$ in the second trimester, and $6.57 \%$ in the third trimester of the participants met the ACOG guidelines during pregnancy (accumulated $\geq 10$ METhours/week, equivalent to 2.5 hours/week of moderateintensity activity; Table 2). Using the Chinese version of the PPAQ, $47.36 \%$ of the score was attributed to the light-intensity activities ( 1.5 to $<3.0 \mathrm{MET}$ ), $40.33 \%$ to sedentary activities ( $<1.5 \mathrm{MET})$, and $12.29 \%$ to moderate-intensity activities ( $\geq 3.0$ to $\leq 6.0 \mathrm{MET}$ ) in the first trimester, which was similar to the other 2 trimesters (Table 3). As to the type of physical activity, $45.15 \%$ of the Chinese version of the PPAQ score was attributed to inactivity, followed by occupation-related activities (33.60\%), household/caregiving activities (13.78\%), transportation-related activities (5.07\%) and sports/ exercise-related activities $(2.45 \%)$ in the first trimester (Table 4). The majority of Chinese women considered slow walking as the most common type of exercise during pregnancy; participation rate ranged from $82.35 \%$ in the first trimester to $90.66 \%$ in the second trimester, followed by stair climbing (63.64\% to $69.39 \%)$. There were only 8 pregnant women engaged in swimming in the first trimester, which reduced to 0 in the third trimester (Table 5).

\section{Discussion}

In this cross-sectional study of 1179 Chinese pregnant women, there were statistically significant differences between meeting ACOG guidelines or not in all the 3 trimesters, respectively, and only $5.63 \%-7.40 \%$ of the pregnant women met the ACOG guidelines (accumulated sports/exercise scores $\geq 10$ MET-hours/week) in the first, second, and third trimesters, respectively. This is consistent with prior observations of lower levels of sports/exercise activities during pregnancy in Americans. ${ }^{18}$ Previous research has shown that most women reduced their physical activity level during the first 20 weeks of pregnancy compared with their level of activity during the year prior to pregnancy, particularly for sports and exercise. ${ }^{19}$ Leiferman and Evenson ${ }^{20}$ reported that the prevalence of meeting physical activity guidelines (defined consistently with ACOG guidelines) was only $12 \%$ among 1979 pregnant women. Differences in study findings are due to variation in the definition of meeting physical activity guidelines as well as racial/ethnic and sociodemographic differences between study samples.

By intensity, the percentage of pregnant women who participated in sedentary and light-intensity activities 


\begin{tabular}{|c|c|c|c|}
\hline Variables & First trimester $(n=391)$ & Second trimester $(n=392)$ & Third trimester $(n=396)$ \\
\hline Age (years) & $29.96 \pm 3.77$ & $30.14 \pm 4.25$ & $29.99 \pm 3.88$ \\
\hline Prepregnancy BMI (kg/m²) & $20.77 \pm 2.94$ & $20.52 \pm 2.78$ & $20.57 \pm 2.53$ \\
\hline \multicolumn{4}{|l|}{ Occupational status } \\
\hline Employed or studying & $295(75.45)$ & $285(72.70)$ & $301(76.01)$ \\
\hline Unemployed & $96(24.55)$ & $107(27.30)$ & 95 (23.99) \\
\hline \multicolumn{4}{|l|}{ Educational status } \\
\hline$<$ High school & $15(3.84)^{*}$ & $33(8.42)^{*}$ & $12(3.03)^{*}$ \\
\hline High school & $134(34.27)$ & $154(39.29)$ & $128(32.32)$ \\
\hline Some college/graduate & $242(61.89)$ & 205 (52.29) & $256(64.65)$ \\
\hline \multicolumn{4}{|l|}{ Annual per capita income $(¥)$} \\
\hline$\leq 24,000$ & $14(3.58)$ & $18(4.59)$ & $10(2.53)$ \\
\hline$>24,000-36,000$ & $48(12.28)$ & $53(13.52)$ & $44(11.11)$ \\
\hline$>36,000$ & $329(84.14)$ & $321(81.89)$ & $342(86.36)$ \\
\hline \multicolumn{4}{|l|}{ Parity } \\
\hline 0 & $303(77.49)$ & 301 (76.79) & $314(79.29)$ \\
\hline$\geq 1$ & $88(22.51)$ & $91(23.21)$ & $82(20.71)$ \\
\hline \multicolumn{4}{|l|}{ Maternal race } \\
\hline Han & $374(95.65)$ & $381(97.19)$ & $387(97.73)$ \\
\hline Minority & $17(4.35)$ & $11(2.81)$ & $9(2.27)$ \\
\hline \multicolumn{4}{|c|}{ Prepregnancy cigarette smoking } \\
\hline No & $382(97.70)$ & 375 (95.66) & $385(97.22)$ \\
\hline Yes & $9(2.30)$ & $17(4.34)$ & $11(2.78)$ \\
\hline \multicolumn{4}{|l|}{ Region of residence } \\
\hline City & $316(80.82)$ & $305(77.81)$ & $336(84.85)$ \\
\hline Countryside & 75 (19.18) & 87 (22.19) & $60(15.15)$ \\
\hline
\end{tabular}

Note: ${ }^{*} p<0.05$.

Table 1. Pregnant women's characteristics.

\begin{tabular}{|c|c|c|c|c|c|}
\hline \multirow{2}{*}{ Gestation scores } & \multicolumn{2}{|c|}{ Accumulated $\geqq 10$ MET-hours/week } & \multicolumn{2}{|c|}{ Accumulated $<10$ MET-hours/week } & \multirow{2}{*}{$p$-value } \\
\hline & $n(\%)$ & Mean \pm SD & $n(\%)$ & Mean \pm SD & \\
\hline First trimester $(n=391)$ & $22(5.63)$ & $344.12 \pm 146.02$ & $369(94.37)$ & $219.14 \pm 72.35$ & 0.000 \\
\hline Second trimester $(n=392)$ & $29(7.40)$ & $297.02 \pm 128.67$ & $363(92.60)$ & $217.41 \pm 72.02$ & 0.000 \\
\hline Third trimester $(n=396)$ & $26(6.57)$ & $304.74 \pm 105.33$ & $370(93.43)$ & $217.59 \pm 79.84$ & 0.037 \\
\hline
\end{tabular}

Table 2. Distribution and scores of sports/exercises according to ACOG guidelines among Chinese pregnant women.

was more than $85 \%$ over the 3 trimesters, and there were few pregnant women engaged in vigorous-intensity activities. The result showed that the intensity of physical activity during pregnancy was too low to meet the ACOG guidelines in majority pregnant women in China. Women with moderate activity showed a $7 \%$ reduction in odds of gestational diabetes mellitus compared with women who reported low activity (odds ratio [OR]: 0.93; 95\% confidence interval [Cl]: 0.72, 1.19). ${ }^{21} \mathrm{~A}$ study conducted in the United States revealed that most obstetricians (74\%) agreed that pregnant women would gain some benefit from moderate-intensity activities. ${ }^{22}$ Yeo et al. ${ }^{23}$ suggested that more vigorous the activities were, the greater the reduction in the risk for preeclampsia. However, other studies have showed that pregnant women reported that light-intensity exercises or physical activities are safer than more vigorous-intensity activities during pregnancy. ${ }^{24,25}$ Therefore, according to the ACOG guidelines, on the assumption of the safety of mothers and fetuses, the Chinese pregnant women should try to 


\begin{tabular}{|c|c|c|c|c|c|c|c|c|c|}
\hline Intensity & Gestation & Mean & SD & Min & 25th & Median & 75th & Max & $p$-value \\
\hline \multirow[t]{3}{*}{ Sedentary } & First trimester & 90.92 & 24.16 & 39.56 & 72.94 & 88.55 & 106.40 & 163.80 & \\
\hline & Second trimester & 83.99 & 23.23 & 42.49 & 65.32 & 79.80 & 99.62 & 159.60 & 0.001 \\
\hline & Third trimester & 87.51 & 24.23 & 29.62 & 69.30 & 82.78 & 101.50 & 163.80 & \\
\hline \multirow[t]{3}{*}{ Light } & First trimester & 106.76 & 53.11 & 1.26 & 75.34 & 107.37 & 133.88 & 292.60 & \\
\hline & Second trimester & 105.95 & 51.06 & 4.19 & 67.74 & 107.59 & 137.18 & 293.10 & 0.753 \\
\hline & Third trimester & 108.16 & 54.68 & 10.14 & 69.54 & 108.28 & 139.13 & 286.55 & \\
\hline \multirow[t]{3}{*}{ Moderate } & First trimester & 27.70 & 39.58 & 0.00 & 4.84 & 10.78 & 39.14 & 334.85 & \\
\hline & Second trimester & 29.50 & 41.44 & 0.00 & 6.00 & 11.40 & 37.43 & 316.24 & 0.047 \\
\hline & Third trimester & 27.13 & 40.40 & 0.00 & 4.84 & 10.82 & 28.31 & 245.76 & \\
\hline \multirow[t]{3}{*}{ Vigorous } & First trimester & 0.06 & 0.29 & 0.00 & 0.00 & 0.00 & 0.00 & 3.01 & \\
\hline & Second trimester & 0.07 & 0.33 & 0.00 & 0.00 & 0.00 & 0.00 & 3.01 & 0.103 \\
\hline & Third trimester & 0.05 & 0.29 & 0.00 & 0.00 & 0.00 & 0.00 & 3.01 & \\
\hline
\end{tabular}

MET, metabolic equivalent; PPAQ, Pregnancy Physical Activity Questionnire.

Table 3. Intensity scores of physical activity levels using the PPAQ among Chinese pregnant women (MET-hours/week)

\begin{tabular}{|c|c|c|c|c|c|c|c|c|c|}
\hline Type & Gestation & Mean & SD & Min & 25th & Median & 75th & Max & $p$-value \\
\hline \multirow[t]{3}{*}{ Inactivity } & First trimester & 101.88 & 29.96 & 41.08 & 79.44 & 99.69 & 119.00 & 189.00 & \multirow{3}{*}{0.010} \\
\hline & Second trimester & 94.29 & 28.31 & 45.86 & 71.20 & 90.52 & 109.40 & 197.40 & \\
\hline & Third trimester & 99.00 & 29.61 & 35.92 & 77.50 & 93.86 & 116.69 & 201.60 & \\
\hline \multirow[t]{3}{*}{ Household/caregiving } & First trimester & 31.10 & 31.57 & 0.00 & 12.43 & 22.64 & 38.15 & 223.36 & \multirow{3}{*}{0.176} \\
\hline & Second trimester & 33.37 & 29.68 & 1.26 & 14.57 & 23.98 & 39.93 & 255.54 & \\
\hline & Third trimester & 31.44 & 28.49 & 3.19 & 13.30 & 22.64 & 40.05 & 216.71 & \\
\hline \multirow[t]{3}{*}{ Occupational } & First trimester & 75.82 & 56.60 & 0.00 & 36.19 & 74.90 & 99.05 & 330.37 & \multirow{3}{*}{0.323} \\
\hline & Second trimester & 77.92 & 60.04 & 0.00 & 32.87 & 74.90 & 101.35 & 364.00 & \\
\hline & Third trimester & 76.10 & 54.00 & 0.00 & 33.57 & 74.90 & 100.71 & 304.36 & \\
\hline \multirow[t]{3}{*}{ Sports/exercise } & First trimester & 5.42 & 9.92 & 0.00 & 1.00 & 4.78 & 5.39 & 115.5 & \multirow{3}{*}{0.001} \\
\hline & Second trimester & 5.63 & 6.46 & 0.00 & 1.38 & 4.94 & 6.00 & 43.27 & \\
\hline & Third trimester & 5.18 & 6.72 & 0.00 & 0.92 & 4.84 & 5.93 & 41.95 & \\
\hline \multirow[t]{3}{*}{ Transportation } & First trimester & 11.44 & 12.41 & 0.00 & 2.10 & 8.75 & 14.49 & 92.75 & \multirow{3}{*}{0.255} \\
\hline & Second trimester & 11.86 & 11.16 & 0.00 & 3.36 & 8.75 & 17.36 & 77.00 & \\
\hline & Third trimester & 11.27 & 13.80 & 0.00 & 2.10 & 8.75 & 14.00 & 124.25 & \\
\hline
\end{tabular}

Table 4. Type scores of physical activity levels using the PPAQ among Chinese pregnant women (MET-hours/week).

participate in some moderate-intensity activities during pregnancy.

By type, only $13.78 \%$ of the PPAQ score was attributed to household/caregiving activities in China. However, household/caregiving activities had been reported to constitute a significant proportion of physical activity during pregnancy in other countries. ${ }^{26}$ Chasan-Taber et al.'s, ${ }^{27}$ collected information on household/caregiving, findings showed that greater amounts of prepregnancy and midpregnancy household/caregiving activities were associated with a decreased risk of gestational diabetes mellitus. This was mainly due to the difference in family culture, since the "one child" policy, the pregnant women rarely engaged in household/caregiving activities. The total types of exercise for pregnant women in China were too unitary, dominated by walking and climbing the stairs. Walking is the most common pattern of physical activity during pregnancy. ${ }^{28}$ In this survey, slow walking could be the most popular activity for women during pregnancy (>82.35\%). In the study by Dempsey et al., ${ }^{5}$ women who reported a brisk usual walking were associated with a lower risk of gestational diabetes. Climbing the stairs and quick walking were also commonly seen among pregnant women. More patterns of exercise should be investigated and provided to pregnant women in the future. 


\begin{tabular}{|c|c|c|c|c|c|c|c|}
\hline \multirow{2}{*}{ Type of exercise } & \multicolumn{2}{|c|}{ First trimester $(n=391)$} & \multicolumn{2}{|c|}{ Second trimester $(n=392)$} & \multicolumn{2}{|c|}{ Third trimester $(n=396)$} & \multirow{2}{*}{$p$-value } \\
\hline & $N$ & $\%$ & $n$ & $\%$ & $N$ & $\%$ & \\
\hline Climbing the stairs & 258 & 65.98 & 272 & 69.39 & 252 & 63.64 & 0.229 \\
\hline Slow walking & 322 & 82.35 & 347 & 88.52 & 359 & 90.66 & 0.001 \\
\hline Quick walking & 95 & 24.30 & 109 & 27.81 & 103 & 26.01 & 0.535 \\
\hline Walking quickly up hills & 30 & 7.67 & 32 & 8.16 & 18 & 4.55 & 0.091 \\
\hline Jogging & 37 & 9.46 & 25 & 6.38 & 17 & 4.29 & 0.014 \\
\hline Prenatal exercise class & 19 & 4.86 & 16 & 4.08 & 8 & 2.02 & 0.089 \\
\hline Swimming & 8 & 2.05 & 3 & 0.77 & 0 & 0.00 & 0.003 \\
\hline Dancing & 25 & 6.39 & 25 & 6.38 & 17 & 4.29 & 0.341 \\
\hline
\end{tabular}

Table 5. Distribution of exercise type in different trimesters using the PPAQ among Chinese pregnant women.

After all, during pregnancy, obstetricians and nurses should provide information to guide pregnant women how to take exercise, and the intensity, duration, and frequency of exercise should start at a level that does not result in pain, shortness of breath, or excessive fatigue. ${ }^{29}$

An important strength of this study is that this is the first large sample survey used for investigating the patterns and intensities of total physical activity during pregnancy (household/caregiving, occupational, sports/ exercise, transportation, and inactivity) in China, which could provide the baseline data for the further study. The study has several limitations. We relied on self-reported measures of physical activity during pregnancy, which may have resulted in misclassification. Future studies should focus on elucidating the use of validated assessment tools and the optimal dose of physical activity during pregnancy for pregnant women, particularly for Chinese pregnant women.

\section{References}

1. Schlüssel $M M$, Souza $E B$, Reichenheim $M E$, et al. Physical activity during pregnancy and maternal-child health outcomes: a systematic literature review. Cadernos de Saude Publica. 2008;24:s531-s544.

2. Streuling I, Beyerlein A, Rosenfeld E, et al. Physical activity and gestational weight gain: a meta-analysis of intervention trials. BJOG Int J Obstet Gynaecol. 2011;118:278-284.

3. Olson CM, Strawderman MS. Modifiable behavioral factors in a biopsychosocial model predict inadequate and excessive gestational weight gain. $J \mathrm{Am}$ Diet Assoc. 2003;103:48-54.

4. Dempsey JC, Sorensen TK, Williams MA, et al. Prospective study of gestational diabetes mellitus risk in relation to maternal recreational physical

\section{Conclusions}

This study represents a group of Chinese women who maintained an inactive lifestyle, including lowintensity activity and unitary type of exercise during their pregnancy. Therefore, obstetricians and midwives should provide the unique opportunity to help their prenatal care patients identify strategies to overcome barriers to adopt and maintain prenatal physical activity.

\section{Acknowledgment}

The authors wish to thank Professor Dong-Tao Lin for copyediting this manuscript.

\section{Conflicts of interest}

All contributing authors declare no conflicts of interest.

activity before and during pregnancy. Am J Epidemiol. 2004;159:663-670.

5. Dempsey JC, Butler CL, Sorensen TK, et al. A case-control study of maternal recreational physical activity and risk of gestational diabetes mellitus. Diabetes Res Clin Pract. 2004;66:203-215.

6. Dempsey FC, Butler FL, Williams FA. No need for a pregnant pause: physical activity may reduce the occurrence of gestational diabetes mellitus and preeclampsia. Exerc Sport Sci Rev. 2005;33:141-149.

7. Bungum TJ, Peaslee DL, Jackson AW, et al. Exercise during pregnancy and type of delivery in nulliparae. $J$ Obstet Gynecol Neonatal Nurs. 2000;29:258-264.

8. ACOG Committee on Obstetric Practice. Committee opinion\# 267: exercise during pregnancy 
and the postpartum period. Obstet Gynecol. 2002;99:171-173.

9. U.S. Department of Health and Human Services. 2008 physical activity guidelines for Americans Washington, D.C. 2008, 1-61. http://www.health. gov/paguidelines. Accessed November 1, 2008.

10. Borodulin K, Evenson KR, Wen F, et al. Physical activity patterns during pregnancy. Med Sci Sports Exerc. 2008;40:1901.

11. Ning Y, Williams MA, Dempsey JC, et al. Correlates of recreational physical activity in early pregnancy. J Matern Fetal Neonatal Med. 2003;13:385-393.

12. Jiang $H, H e G$, Li M, et al. Reliability and validity of a physical activity scale among urban pregnant women in eastern China. Asia Pac J Public Health, 2015;27:1208-1216.

13. Wang $\mathrm{YL}$, Zhao $\mathrm{XH}$. Occupational health of working women in China. Asia-Pac J Public Health. 1987;1:66-71.

14. Center for Health Statistics and Information, Ministry of Health. An Analysis Report of National Health Services Survey in China, 2008. Beijing, China: China Xiehe Medical University; 2009:409.

15. Chasan-Taber L, Schmidt MD, Roberts DE, et al. Development and validation of a pregnancy physical activity questionnaire. Med Sci Sports Exerc. 2004;36:1750-1760.

16. Schmidt MD, Pekow P, Freedson PS, et al. Physical activity patterns during pregnancy in a diverse population of women. J Womens Health. 2006;15:909-918.

17. Ainsworth BE, Haskell WL, Leon AS, et al. Compendium of physical activities: classification of energy costs of human physical activities. Med Sci Sports Exerc. 1993;25:71-80.

18. Chasan-Taber L, Fortner RT, Gollenberg A, et al. A prospective cohort study of modifiable risk factors for gestational diabetes among Hispanic women: design and baseline characteristics. J Women's Health. 2010;19:117-124.

19. Fell DB, Joseph KS, Armson BA, et al. The impact of pregnancy on physical activity level. Matern Child Health J. 2009;13:597-603.

20. Leiferman JA, Evenson KR. The effect of regular leisure physical activity on birth outcomes. Matern Child Health J. 2003;7:59-64.

21. Redden SL, LaMonte MJ, Freudenheim JL, et al. The association between gestational diabetes mellitus and recreational physical activity. Matern Child Health J. 2011;15:514-519.

22. Evenson KR, Pompeii LA. Obstetrician practice patterns and recommendations for physical activity during pregnancy. J Womens Health. 2010;19:1733-1740.

23. Yeo SA, Davidge S, Ronis DL, et al. A comparison of walking versus stretching exercises to reduce the incidence of preeclampsia: a randomized clinical trial. Hypertens Pregnancy, 2008;27:113-130.

24. Mudd LM, Nechuta S, Pivarnik JM, et al. Factors associated with women's perceptions of physical activity safety during pregnancy. Prev Med. 2009;49:194-199.

25. Duncombe D, Wertheim EH, Skouteris H, et al. Factors related to exercise over the course of pregnancy including women's beliefs about the safety of exercise during pregnancy. Midwifery. 2009;25:430-438.

26. Chasan-Taber L, Schmidt MD, Pekow P, et al. Correlates of physical activity in pregnancy among Latina women. Matern Child Health J. 2007;11:353-363.

27. Chasan-Taber L, Schmidt MD, Pekow P, et al. Physical activity and gestational diabetes mellitus among Hispanic women. J Womens Health. 2008;17:999-1008.

28. Zhang J, Savitz DA. Exercise during pregnancy among US women. Ann Epidemiol. 1996;6:53-59.

29. Wang TW, Apgar BS. Exercise during pregnancy. Am Fam Phys. 1998;57:1846-1859. 